
allemande

47-1| 2015

L'Allemagne et l'Europe | La déprise de l'Empire napoléonien en Allemagne en 1813

\title{
Entre opposition et intégration. Les départements du Rhin dans la première phase de la restauration (1814-1832)
}

Gabriele B. Clemens

\section{OpenEdition}

\section{Journals}

Édition électronique

URL : https://journals.openedition.org/allemagne/469

DOI : 10.4000/allemagne.469

ISSN : 2605-7913

Éditeur

Société d'études allemandes

Édition imprimée

Date de publication : 26 juin 2015

Pagination : 151-162

ISSN : 0035-0974

Référence électronique

Gabriele B. Clemens, «Entre opposition et intégration. Les départements du Rhin dans la première phase de la restauration (1814-1832) ", Revue d'Allemagne et des pays de langue allemande [En ligne], 47-1 | 2015, mis en ligne le 13 décembre 2017, consulté le 22 mai 2021. URL : http:// journals.openedition.org/allemagne/469; DOI : https://doi.org/10.4000/allemagne.469 


\title{
Entre opposition et intégration. Les départements du Rhin dans la première phase de la restauration (1814-1832)
}

\author{
- Gabriele B. Clemens*
}

En vingt ans seulement - de 1794 à 1814 - la rive gauche du Rhin, zone frontière entre France et Allemagne, connut deux changements de régime à la suite de la Révolution française. Auparavant, le territoire était émietté entre une multitude de possessions ecclésiastiques ou laïques, dominées par les trois archevêchés et électorats de Mayence, Trèves et Cologne. Puis venaient les vastes possessions du Palatinat, des principautés de Nassau-Sarrebruck, des Deux-Ponts (au sud) et de la baronnie Juliers (au nord-ouest). Grâce à la succession de l'ancienne baronnie de Clèves, la Prusse avait aussi un pied sur le Rhin inférieur depuis le XVIII ${ }^{\mathrm{e}}$ siècle. Les villes libres impériales d'Aix-la-Chapelle et de Cologne avaient pu conserver leur rang. Une mosaïque de comtés et de principautés les entouraient ${ }^{(1)}$. Autoritaires ou éclairés, tous ces gouvernements ou presque échouèrent à réformer leurs États, à cause des résistances considérables auxquelles ils se heurtaient ${ }^{(2)}$. Cologne et Aix ne parvinrent pas non plus à secouer le carcan des privilèges, malgré l'intensité des affrontements entre réformateurs et traditionalistes ${ }^{(3)}$.

La Révolution qui ébranla en 1789 la France dans ses fondements fit sentir ses effets jusque dans la Rhénanie voisine. Des affiches, des pamphlets appelèrent à résister contre les princes ${ }^{(4)}$. Puis le calme revint et ce n'est qu'à la déclaration de guerre

* Professeur d'histoire moderne à l'Université de la Sarre.

1 Franz Irsigler, Herrschaftsgebiete im Jahr 1789, Cologne, Rheinland Verlag, 1982.

2 Sur le dernier prince électeur de Trèves, voir la récente parution de Gabriele B. Clemens, "Clemens Wenzeslaus: Trierer Kurfürst im europäischen Kontext », in: Michael Емвасн, Reinhold Boнlen (dir.), Der Trierer Erzbischof und Kurfürst Clemens Wenzeslaus (1739-1812) - Eine historische Bilanz nach 200 Jahren, Mayence, Selbstverlag der Gesellschaft für Mittelrheinische Kirchengeschichte e.V., 2014, p. 3-21.

3 Michael Rowe, From Reich to State. The Rhineland in the Revolutionary Age 1780-1830, Cambridge, Cambridge University Press, 2003.

4 Günther BIRTsch, «Soziale Unruhen, ständische Repräsentation. Trier in der Zeit der Französischen Revolution ", in: Mentalitäten und Lebensverhältnisse, Göttingen, Vandenhoeck \& Ruprecht, 1982, p. 143-159; Johannes Schmitт, Revolutionäre Saarregion 1789-1850, St. Ingbert, Röhrig, 2005. 
austro-prussienne que les pays rhénans furent entraînés au cœur des événements politiques. En 1794, ils furent conquis par les troupes françaises. Les premières années, des administrations rivales, militaires et civiles, se succédèrent à un rythme rapide ${ }^{(5)}$. La population souffrit du passage des troupes et des frais considérables occasionnés par leur entretien; on était bien loin de liberté, égalité, fraternité( ${ }^{(6)}$ ou de "guerre aux palais, paix dans les chaumières ». Puis en octobre 1797, dans les articles secrets de la paix de Campo Formio, l'Autriche renonça à toute la rive gauche du Rhin; dès lors l'administration commença à être calquée sur le modèle français. Plusieurs mesures en jetèrent les bases : réorganisation de la bureaucratie et de la justice (par le commissaire François Joseph Rudler, conformément aux instructions du Directoire); introduction des procédures judiciaires françaises; liberté du commerce et de l'artisanat (fin des corporations); réforme des impôts et suppression des privilèges, donc de la dîme. La disparition des frontières à l'ouest ouvrit au commerce un marché impérial de vingt-cinq millions de consommateurs. L’année 1798 fit époque en Rhénanie: presque tous les domaines furent touchés par les réformes, partout la nouveauté remplaçait les vieilles traditions ${ }^{(7)}$. Cette rupture radicale avec des privilèges obsolètes, l'égalité des citoyens devant la loi, l'introduction d'un appareil juridique moderne et la liberté d'entreprendre créèrent les conditions nécessaires au développement de la région. Pour la première fois en territoire allemand, les juifs furent complètement émancipés ${ }^{(8)}$. À partir du 9 février 1801 (paix de Lunéville), les mêmes lois qu'en France furent appliquées aux départements de la rive gauche du Rhin. Le changement de pouvoir ne rencontra aucune résistance: les anciennes élites travaillèrent avec les nouvelles, aucun soulèvement ne se produisit, contrairement au Luxembourg voisin. Après 1815, les notables rhénans cherchèrent par tous les moyens à conserver les acquis de 1798 et le code Napoléon.

Lorsque les troupes de la Coalition franchirent le Rhin au début de 1814 pour continuer vers l'ouest, les territoires allemands de la rive gauche se trouvaient dans un état bien différent de l'Ancien Régime. Les " troupes de libération » ne furent pas reçues avec des transports d'enthousiasme (de quoi devait-on être libéré?) pas plus qu'il n'y eut de chasse aux Français pourtant vaincus, comme le souligne Ute Planert dans son livre sur le mythe des guerres de libération ${ }^{(9)}$. La population, inquiète, se tint

5 Jacques Godechot, Les Commissaires aux armées sous le Directoire. Contribution à l'étude des rapports entre les pouvoirs civils et militaires, Paris, Fustier, 1941; Max BRAUBACH, «Vom Westfälischen Frieden bis zum Wiener Kongreß (1648-1815) », in: Franz Petri, Georg Droege (dir.), Rheinische Geschichte, Düsseldorf, Schwann, 1976, p. 219-365; Timothy Charles William Blanning, The French Revolution in Germany. Occupation and Resistance in the Rhineland 1792-1802, Oxford, Clarendon, 1983. Blanning exagère en considérant que la population eut une attitude de résistance envers les Français, alors qu'elle était avant tout passive et que dans certains cercles intellectuels régna une atmosphère d'enthousiasme révolutionnaire lorsque les Français jetèrent à bas les vieilles structures de l'Ancien Régime.

6 Les passages en italiques sont en français dans le texte (NDT).

7 Jürgen MüLlER, « 1798 - Das Jahr des Umbruchs im Rheinland », Rheinische Vierteljahrsblätter, 62 (1998), p. 205-237.

8 Cilli Kasper-Holtкотte, Juden im Aufbruch. Zur Sozialgeschichte einer Minderheit im Saar-MoselRaum um 1800, Hanovre, Hahn, 1996.

9 Ute Planert, Der Mythos vom Befreiungskrieg. Frankreichs Kriege und der deutsche Süden, Paderborn, Schöningh, 2007. Pierre Horn, dans sa thèse sur «Le Défi de l'enracinement napoléonien entre 
tranquille. Lorsque le 5 janvier 1814, l'avant-garde de l'armée prussienne commandée par Henckel von Donnersmarck arriva sous les murs de Trèves, la cour d'appel continua de travailler comme si de rien n'était. Le grand-maître de la loge maçonnique, le procureur général lorrain Georges François Bidault, faisait visiblement entièrement confiance à la fraternité franc-maçonne car la dernière séance qu'il présida comptait déjà des officiers prussiens parmi les participants ${ }^{(10)}$.

Pour les Rhénans qui soupiraient devant les frais d'entretien des troupes coalisées, les mois qui suivirent furent pleins d'incertitude. Que deviendraient leurs États après le retrait français? Les idées les plus folles circulaient. Personne ne pensait sérieusement au retour des princes enfuis. L'archevêque de Trèves, Clemens Wenzeslaus, était mort; la branche des Nassau de Sarrebruck, éteinte. En revanche, les aristocrates des cités jadis libres spéculaient sur la restauration de leur pouvoir. Le comte Edmund von Kesselstatt se rendit à Vienne en 1814 et 1815, tout d'abord au nom de sa famille, puis de la noblesse rhénane et luxembourgeoise, avec moult demandes d'indemnités pour les droits et privilèges perdus. Malgré ses efforts infatigables, il n'eut pas gain de cause. L'acte de naissance de la Confédération germanique rejeta en bloc les revendications des anciens chevaliers d'Empire (article 14) ${ }^{(11)}$.

On évoqua même au congrès de Vienne en 1815 l'idée de "transplanter » sur les bords du Rhin les Wettin, rois de Saxe, afin de laisser la Saxe à la Prusse. Cette dernière avait l'ambition d'absorber la Saxe dont le roi était resté trop longtemps allié à Napoléon à son goût; mais l'Angleterre et l'Autriche s'interposèrent pour éviter que la Prusse ne prenne trop de place en Allemagne centrale. En compensation, elle reçut des terres en Rhénanie et en Westphalie dont elle n'avait que faire. Les autres puissances espéraient que la présence de troupes prussiennes sur le Rhin empêcherait la France de recommencer à s'étendre vers l'est ${ }^{(12)}$.

Nous allons nous concentrer ici sur les territoires accordés à la Prusse sur la rive gauche du Rhin par le congrès de Vienne, le 8 février 1815: l'ancien département Rhin et Moselle, ainsi qu'une partie des départements de l'Ourthe, de la Meuse inférieure, de la Roër, de la Sarre et des Forêts, le tout progressivement désigné sous le nom de " province rhénane» (nom officiel à partir de 1830 ${ }^{(13)}$. Cette région est caractérisée par

Rhin et Meuse (1810-1814). Étude transnationale de l'opinion publique dans les départements de la Roër (Allemagne), de l'Ourthe (Belgique), des Forêts (Luxembourg) et de la Moselle (France) » (Paris IV, 2013), parvient au même résultat. La publication de la thèse est prévue pour 2015.

10 Winfried Dotzauer, «Das aufgeklärte Trier. Freimaurer und Lesegesellschaft bis zum Ende der napoleonischen Zeit ", in: Johannes BÄrlich, Alois Gerlich, Ludwig Petry (dir.), Geschichtliche Landeskunde, 9, Wiesbaden, Steiner, 1973, p. 246. Sur Bidault, voir Gabriele B. Clemens, « Die Notabeln der Franzosenzeit », in: Elisabeth DüHr, Christl LehnerT-Leven (dir.), Unter der Trikolore. Sous le drapeau tricolore. Trier in Frankreich - Napoleon in Trier. Trèves en France - Napoléon à Trèves, Trèves, Städtisches Museum Simeonstift, 2004, p. 113-114.

11 Christof Dipper, «Der Rheinische Adel zwischen Revolution und Restauration », in: Helmut Feigel, Willibald Rosener (dir.), Adel im Wandel. Vorträge und Diskussionen des elften Symposions des Niederösterreichischen Instituts für Landeskunde Horn, 2.-5.7.1990, Vienne, Nieder-Österreichisches Institut für Landeskunde, 1991, p. 91-112.

12 Reinhard Stauber, Der Wiener Kongress, Stuttgart, UTB, 2014.

13 Rüdiger Sснüтz, Preußen und die Rheinlande. Studien zur preußischen Integration im Vormärz, Wiesbaden, Steiner, 1979, p. 27. 
un nombre de conflits nettement plus élevé que dans le Palatinat et à Mayence où les nouveaux maîtres, respectivement bavarois et hessois, furent mieux accueillis car leur État était plus libéral. Sur la rive gauche devenue prussienne, l'enthousiasme était très modéré. Trois points névralgiques concentrèrent les tensions entre la restauration de 1814 et le début des années 1830 : la politique prussienne envers les fonctionnaires, la question des biens nationaux et la querelle sur la participation politique.

Après de chaotiques premières années sous l'administration militaire française, la majorité des bureaucrates avait accepté l'autorité française pour la bonne raison qu'elle était plus rationnelle. La pratique d’Ancien Régime avait été pesamment aristocratique et collégiale; la nouvelle administration apportait une organisation juridique adaptée à l'époque, une répartition claire des compétences et des procédures nettement modernisées ${ }^{(14)}$. Dans un premier temps, les nouveaux maîtres prussiens eurent l'intelligence de conserver une administration qui avait fait ses preuves, d'autant plus qu'ils avaient besoin de gens compétents puisqu'ils conservaient des Français le droit et l'administration des domaines. On remplaça simplement les termes français par des allemands: un receveur des domaines devint un Einnehmer, un maire fut appelé Bürgermeister. La grande majorité des fonctionnaires poursuivit sa carrière, occupant les places laissées vacantes (surtout dans l'administration financière et forestière) par leurs collègues français qui avaient presque tous quitté le pays. Ceci permit aux autochtones de monter en grade; on fit aussi venir de nouveaux employés de la rive droite du Rhin. Mais les postes de direction des districts et de la province furent confiés à des Prussiens, ce qui souleva tout de suite des protestations et tendit sérieusement les relations avec les Rhénans.

Les fonctionnaires français demandant leur intégration à l'administration allemande ne l'obtinrent pas, comme Joseph Massa de Strasbourg, ancien inspecteur des forêts à Trèves ${ }^{(15)}$. En revanche, les Allemands furent rarement inquiétés pour leurs sympathies françaises ${ }^{(16)}$. Comme ils l'avaient fait à l'époque française, presque aucun ne montrait de regret à servir un nouveau maître; beaucoup changeaient de régime pour la troisième fois. Ainsi le juriste Johann Heinrich Rosbach garda-t-il après 1815 son poste au tribunal d'appel de Trèves, puis il fut nommé conseiller régional du tribunal à Trèves en 1820 après que le tribunal eut été transféré à Cologne en 1819; ses émoluments se montaient alors à mille thalers mensuels. Le gouvernement prussien ne

14 Gabriele B. Clemens, "Diener dreier Herren - die Beamtenschaft in den linksrheinischen Gebieten vom Ancien Regime bis zur Restauration ", in: Helga Schnabel-Schüle, Andreas Gestrich (dir.), Fremde Herrscher - fremdes Volk. Inklusions- und Exklusionsfiguren bei Herrschaftswechseln in Europa, Trèves, Lang, 2006, p. 73-102.

15 Un dossier sur Massa se trouve aux archives régionales de Coblence, fonds 354-523.

16 Jakob Christian Schmeltzer, fonctionnaire des impôts, fut certes enfermé à Düsseldorf pour ses sympathies bonapartistes, mais il fut libéré après cinq semaines de prison et entièrement réhabilité. Quelques années plus tard, il devint conseiller de la province et du gouvernement. D’après son biographe, on lui aurait reproché son épouse française, son personnel de la même nationalité, le fait qu'il parle mieux français qu'allemand et surtout son amitié avec le dernier préfet du département de la Sarre, le baron Alexandre-François Bruneteau de Sainte-Suzanne. À l'heure actuelle, on ne dispose pas de recherches systématiques sur les fonctionnaires que les nouvelles autorités accusèrent de sympathie pour le régime napoléonien. Sur Schmeltzer, voir Carl Bittmann, « Jacob Christian Schmeltzer und die Achard'sche Departements-Zuckerfabrik im St. Agnetenkloster zu Trier, Anno 1811-14 », Trierisches Archiv, 2 (1901), p. 1-137. 
vit pas non plus d'inconvénient à lui reconnaître les mérites acquis dans l'administration française; lors du cinquantième anniversaire de son entrée dans le service public, le roi de Prusse lui remit l'ordre de l'Aigle rouge (troisième classe), alors que Rosbach n'était fonctionnaire prussien que depuis quatorze ans ${ }^{(17)}$.

Ces fonctionnaires qui passaient d'un régime à l'autre s'étaient mis au service de l'État pour assurer leur situation matérielle, mais aussi par sentiment de responsabilité et parfois, pour ceux entrés pendant la dernière décennie du XVIII ${ }^{\mathrm{e}}$ siècle, par sympathie pour les idées révolutionnaires. Vers 1800, l'argument national ou le sentiment d'une domination étrangère semblent n'avoir joué qu'un rôle secondaire sur la rive gauche du Rhin. Karl-Georg Faber, auteur en 1960 d'une étude sur la fonction publique et les Français en Rhénanie, le résumait ainsi: "Ces fonctionnaires, comme la majorité de la population, n'avaient alors aucune conscience nationale ${ }^{(18)}$. Depuis, la recherche a confirmé cette conclusion dans ses grandes lignes ${ }^{(19)}$. Cet état d'esprit facilita sans doute l'arrivée du nouveau régime et de ses administrateurs. Le mythe national du Rhin romantique, produit des guerres de libération, n'est pas né sur les bords du Rhin. Ses chantres tardifs (il atteint son apogée vers 1840) n'étaient pas des Rhénans.

En 1814, le changement de maître ne provoqua pas non plus d'opposition sérieuse, sauf exception. Après l'entrée des coalisés, Georg Friedrich von Rebmann, vice-président de la cour d'appel à Trèves, resta d'abord indécis; devait-il entrer au service du roi de Prusse ou non? Convaincu de ne pouvoir vivre et travailler que là où ses idéaux seraient mis en œuvre, il décida de s'installer dans le Palatinat bavarois où, pensait-il, l'héritage de la Révolution serait conservé, tandis que la Rhénanie prussienne risquait de retourner en arrière ${ }^{(20)}$. Ses collègues n'eurent pas ces scrupules, ou bien ces derniers

17 Constantin Cnyrim, « Die Trierer Familie Rosbach », Kurtrierisches Jahrbuch, 25 (1985), p. 221-235.

18 Karl-Georg FABER, "Verwaltungs- und Justizbeamte auf dem linken Rheinufer », in: Max BRAUBACH (dir.), Geschichte und Landeskunde. Festschrift für Franz Steinbach, Bonn, Röhrscheid, 1960, p. 367.

19 Indiquons ici les travaux de Hansgeorg Molitor, Vom Untertan zum Administré. Studien zur französischen Herrschaft und zum Verhalten der Bevölkerung im Rhein-Mosel-Raum von den Revolutionskriegen bis zum Ende der napoleonischen Zeit, Wiesbaden, Steiner, 1980; d'Uwe Andrae, Die Rheinländer, die Revolution und der Krieg 1794-1798: Studie über das Erzstift Köln unter der Besatzung durch französische Revolutionstruppen 1794-1798 im Spiegel von Petitionen, Essen, Klartext-Verlag, 1994. Winfried Speitkamp approfondit ces recherches en démontrant que pendant la période napoléonienne, les protestations en Allemagne étaient surtout dirigées contre le poids de l'État moderne, notamment de la bureaucratie, mais pas expressément anti-françaises. Leurs formes comme leur contexte d'apparition appartiennent plutôt à la tradition protestataire de l'époque moderne. Les résistances à la conscription et les désertions n'étaient pas liées non plus spécifiquement à la présence de l’armée française. Voir Winfried SPEITKAMP, "Sozialer und politischer Protest im napoleonischen Deutschland», in: Walter Heinemeyer (dir.), Hundert Jahre Historische Kommission für Hessen; 1897-1997, Marbourg, Elwert, 1997, p. 713-730.

20 Cet homme de plume et juriste éclairé, défenseur passionné des idéaux de 1789, fit carrière sous Napoléon dans l'appareil judiciaire. En 1816, on le nomma président du tribunal d'appel à Deux-Ponts, dans le territoire attribué à la Bavière sur les bords du Rhin; en 1818, il fut anobli. Au soir de sa vie (il mourut à l'âge encore jeune de cinquante-six ans), il réussit à faire conserver le droit français dans le Palatinat. Sa renommée, portée par ses écrits théoriques sur le droit français et ses articles politiques, dépassa les frontières de la province. Il fut l'un des soutiens les plus énergiques du libéralisme, sans aucune concession au nationalisme romantique qui pointait après 1815. Voir Karl Georg FABER, «Die Übernahme von A.G.F. Rebmann in den bayerischen Dienst ", Mitteilungen des Historischen Vereins für die Pfalz, 56 (1958), p. 152-157; Elmar WAdLE, Gerhard SAuder (éd.), Georg Friedrich Rebmann (1768-1824): Autor, Jakobiner, Richter, Sigmaringen, Thorbecke, 1997. 
ne suffisaient pas pour l'imiter: ils s'adaptèrent à leurs nouveaux maîtres comme aux précédents ${ }^{(21)}$.

Mais cela n'impliquait pas de renoncer aux droits acquis ni à l'administration moderne dans ses principes. La Prusse voulait aligner l'administration et la justice des territoires reçus à Vienne sur ses lois; mais elle y renonça devant les résistances. Les cadres juridiques et judiciaires demeurèrent quasi-inchangés. On ne toucha pas non plus à l'organisation communale; les communes urbaines et rurales restèrent à égalité de droits. Les notables rhénans défendirent avec véhémence ces acquis pour lesquels ils forgèrent l'expression d'« institutions rhénanes ». Entre 1820 et 1848, cette opposition à l'administration prussienne, civile et militaire, se manifesta à plusieurs reprises dans certains des cercles de sociabilité (Kasinogesellschaften) fondés dans les grandes villes autour de 1800. Dans le Palatinat, la Bavière se heurtait à la même résistance que la Prusse. En 1832, la fête de Hambach en Palatinat donna lieu à la plus grande manifestation libérale et démocratique d'avant 1848. On estime le nombre des participants entre vingt et trente-deux mille; ils vinrent non seulement du Palatinat, mais aussi des pays voisins et de Prusse ${ }^{(22)}$. Transformer les Français en ennemis héréditaires prit donc des décennies; ce n'est que tardivement que la domination française fut considérée comme une domination étrangère.

En revanche, des rumeurs affolantes sur le retour de la féodalité couraient dans les territoires reconquis par la Prusse. En 1801, la paix de Lunéville avait donné la rive gauche du Rhin à la France, apparemment pour toujours. Dès lors, les biens de l'Église et de la noblesse qu'on avait déjà confisqués avaient été vendus comme en France sous la Révolution. Ces mesures de sécularisation ou de médiatisation provoquèrent, entre 1803 et 1813, un transfert de richesse d'une ampleur jusqu'alors inconcevable. Certes, des mesures de sécularisation avaient été prises dans certains États de la rive droite du Rhin après le recès de la Diète d'Empire de 1803, mais sans aller jusqu'à vendre les biens de l'Église, mis à part en Bavière ${ }^{(23)}$. Les territoires de la rive gauche du Rhin, maintenant sous administration française, furent touchés par ce genre de mesures d'une façon beaucoup plus radicale. Entre 1803 et 1813, on y vendit presque quatorze mille biens nobles ou ecclésiastiques dits « biens nationaux » qui représentaient environ $12 \%$ de la surface cultivable de chaque département. Leur adjudication rapporta à l'État plus de soixante-six millions de francs ${ }^{(24)}$.

21 Manfred Koltes, Das Rheinland zwischen Preußen und Frankreich. Studien zu Kontinuität und Wandel am Beginn der preußischen Herrschaft (1814-1822), Cologne, Böhlau, 1992, p. 488.

22 Cornelia Förster, Der Press- und Vaterlandsverein von 1832/33. Sozialstruktur und Organisationsformen der bürgerlichen Bewegung in der Zeit des Hambacher Festes, Trèves, Trierer historische Forschungen, 1982; Joachim Kermann (dir.), Freiheit, Einheit und Europa: Das Hambacher Fest von 1832 - Ursachen, Ziele, Wirkungen, Ludwigshafen, pro MESSAGE, 2006.

23 L'article de Christof Dipper fait référence à la sécularisation en Allemagne: Christof DiPPER, " Probleme einer Wirtschafts- und Sozialgeschichte der Säkularisation in Deutschland (1803-1813) », in: Armgard von Reden-Dohna (dir.), Deutschland und Italien im Zeitalter Napoleons, Wiesbaden, Steiner, 1979, p. 123-170.

24 Wolfgang Schieder (dir.), Säkularisation und Mediatisierung in den rheinischen Departements: 1803 1813. Edition des Datenmaterials der zu veräußernden Nationalgüter, 7 volumes, Boppard am Rhein, Harald Boldt Verlag, 1991. 
Ces ventes se faisaient au seul profit du Trésor public. L’objectif premier était de vendre beaucoup de terres le plus rapidement possible. Une réforme sociale par des redistributions agraires (ce que visaient les ventes du début de la Révolution) n'était plus à l'ordre du jour sous Napoléon. Cette politique eut des répercussions considérables sur la classe des marchands: ceux de la ville furent surreprésentés alors qu'il ne s'agissait pas d'immobilier urbain, mais de terres et de domaines agricoles, car ils avaient rapidement compris le profit à tirer de ce nouveau marché. À côté de l'État français, c'est ce groupe de notables qui profita le plus de la vente des biens nationaux. Ils purent conforter leur position économique et sociale, voire l'augmenter considérablement par la suite puisque, pendant tout le $\mathrm{XIX}^{\mathrm{e}}$ siècle, les droits politiques en Prusse dépendirent de la propriété foncière ${ }^{(25)}$.

Après le départ des Français, on s'inquiéta beaucoup de savoir si les ventes des biens nationaux allaient être déclarées juridiquement nulles. Ces craintes transparaissent dans une lettre de juin 1814 envoyée par Damian Cardon, acheteur d'immobilier et ancien conseiller à la cour de Trèves, au chancelier Johann von Hügel, son ancien supérieur: "La nouvelle de la paix conclue avec la France se confirme et, pour beaucoup, d'une façon fort agréable puisqu'il est écrit que les aliénations des Domaines faites par la France dans votre Pays qui est cédé, sont maintenues en vertu du traité de paix »(26).

Mais les rumeurs continuèrent de circuler dans les années suivantes, comme on peut le voir dans cette lettre du ministère secret d'État royal au gouvernement général du Bas-Rhin en mai 1815. Un homme bien connu aurait rapporté la crainte " qu'en effet, 1. les achats de biens nationaux p[uiss]ent être attaqués par la suite, alors que plus de la moitié de la population avait participé à ces ventes, soit comme acheteur, soit comme créancier sur hypothèque; 2 . qu'en changeant le système des impôts, on réintroduise une dîme d'État " ${ }^{(27)}$. Cette lettre anonyme exagère la proportion des Rhénans engagés dans de telles affaires, mais elle reflète l'inquiétude générale. C'est seulement le 9 mars 1819 que les achats de l'époque française furent formellement reconnus par un ordre du cabinet royal ${ }^{(28)}$.

25 Bernard Bodinier, Éric Teyssier, L’événement le plus important de la Révolution. La vente des biens nationaux (1789-1867) en France et dans les territoires annexés, Paris, Société des Études Robespierristes, 2000; sur les départements de la rive gauche du Rhin, cf. Michael MüLler, Säkularisation und Grundbesitz. Zur Sozialgeschichte des Saar-Mosel-Raumes 1794-1813, Boppard am Rhein, Harald Boldt, 1980; Gabriele B. Clemens, Immobilienhändler und Spekulanten. Die sozial- und wirtschaftsgeschichtliche Bedeutung der Großkäufer in den rheinischen Departements, Boppard am Rhein, Harald Boldt Verlag, 1995; G.B. Clemens, « Napoleonische Armeelieferanten und die Entstehung des Rheinischen Wirtschaftsbürgertums », Francia, 24/2 (1997), p. 159-180; G.B. Clemens, " Gros acheteurs et spéculateurs dans les ventes aux enchères de biens nationaux dans les départements rhénans à l'époque napoléonienne ", Annales Historiques de la Révolution française, 70 (1998), p. 669-674; G.B. Clemens, «Besitzumschichtungen im Rheinland aufgrund der Nationalgüterauktionen (18031813) ", in: Georg MöLICH, Joachim Oepen, Wolfgang Rosen (dir.), Klosterkultur und Säkularisation im Rheinland, Essen, Klartext-Verlag, 2002, p. 331-349.

26 Karl ZimmermanN, "Stimmungsberichte des ehemaligen Kurtrierischen Hofrates Damian Cardon zur politischen Neugestaltung in den Jahren 1814 und 1815 », Trierisches Jahrbuch, 8 (1957), p. 57.

27 Archives principales d'État de Düsseldorf, fonds GGNM 275, Biens domaniaux et de dotation, lettre du 12 mai 1815.

28 Archives régionales de Coblence, fonds 441-9068. 
Il n'y a pas d'évaluation précise sur la quantité de biens nationaux allés à l'État prussien, mais elle était sans doute importante, malgré les ventes antérieures, comme l'attestent les ventes aux enchères faites par le gouvernement à partir de 1818 . Rien que dans le district de Coblence, plus de sept cents biens furent proposés à la vente, dont cent trente domaines de grande valeur; huit cent cinquante dans l'ancien département de la Roër. À première vue, les modalités de cession ne se distinguaient guère des françaises; ainsi les forêts domaniales devaient-elles rester entières. En revanche, les évaluations furent revues à la hausse et, en conséquence, l'enchère de départ aussi, tandis que les délais de paiement étaient drastiquement réduits: les guerres avaient vidé les caisses et l'État prussien avait besoin d'argent au plus vite ${ }^{(29)}$.

Un mot encore sur la noblesse rhénane. Si l'on ne dispose pas encore d'études de fond sur elle avant 1848, l'état de l'historiographie permet néanmoins de supposer que la noblesse rhénane catholique garda ses distances par rapport à la maison royale protestante et demeura dans sa province ${ }^{(30)}$. En Prusse, Frédéric le Grand avait comblé de privilèges la noblesse militaire et d'État. Après les catastrophes d'Iéna et d'Auerstedt, elle était tombée un temps en disgrâce, mais la Restauration lui avait redonné la prééminence pour pourvoir les postes de commandement. En Rhénanie, la noblesse ne put imposer le retour à la féodalité, mais elle réussit à faire supprimer le partage égalitaire des héritages. Le roi, par un ordre du cabinet de 1826, rétablit l'hérédité de la noblesse dans les territoires rhénans ${ }^{(31)}$. La même année, majorats et fidéicommis furent de nouveau autorisés. À temps pour la première réunion de la diète provinciale, Berlin émit un ordre qui rendait caduques toutes les lois françaises ayant supprimé les titres, désignations et armes de la noblesse. Enfin, même si le retour à la féodalité s'arrêta là, la noblesse fut privilégiée dans la représentation politique (déjà très restreinte) d'une façon inenvisageable pour les notables bourgeois.

Les débats sur la participation politique et la constitution étaient intimement liés aux controverses sur l'héritage français. Les années 1813-1815 avaient été ainsi fertiles en rumeurs sur ce sujet, avant que l'annexion prussienne ne fasse émerger de la cacophonie des positions plus nettes, dont aucune n'était démocrate ou républicaine. Les décrets de Carlsbad en 1819 mirent de toute façon un terme aux discussions politiques, puisque la censure fut renforcée et les publications réclamant plus de droits politiques, interdites. Auparavant, entre 1813 et 1819, les discussions tournèrent autour des promesses de constitution faites dans divers textes: les deux patentes du 5 avril 1815 sur la prise de possession du Rhin, l'ordonnance du 22 mai 1815 de Frédéric Guillaume III sur la représentation du peuple, l’article 13 de la Confédération

29 G. B. Clemens, Immobilienhändler und Spekulanten (note 25), p. 225-236.

30 On ne dispose actuellement que de travaux partiels: Reinhold K. Weitz, « Die preußische Rheinprovinz als Adelslandschaft. Eine statistische, sozialgeschichtliche und kulturräumliche Untersuchung zum frühen 19. Jahrhundert », Rheinische Vierteljahrsblätter, 38 (1974), p. 333-354; C. DipPER, " Der Rheinische Adel zwischen Revolution und Restauration » (note 11); Gabriele B. Clemens, "Wie Phoenix aus der Asche? Der rheinische Adel zur Zeit Napoleons ", in: Jacques Olivier Boudon, Gabriele B. Clemens, Pierre Horn (dir.), Franzosen und Deutsche im napoleonischen Empire: Konsens, Kollaboration oder Konfrontation?, Munich, Oldenbourg (sous presse).

31 Christof Dipper, «Die Reichsritterschaft in napoleonischer Zeit », in: Eberhard Weis (dir.), Reformen im rheinbündischen Deutschland, Munich, Oldenbourg, 1984, p. 60. 
germanique décidé au congrès de Vienne le 8 juin, d'après lequel quelques États allemands avaient droit à une constitution. Un ordre du cabinet royal du 30 mars 1817 promettait de garantir la représentation du peuple dans les provinces comme dans l'ensemble de l'État prussien dans un avenir proche ${ }^{(32)}$. Qu'est-ce que le roi de Prusse entendait par "proche ", se demandait-on? Comment définir ce que devait être une représentation adéquate du peuple?

Comme nous l'avons dit, les discussions sur la constitution, encore possibles en Rhénanie entre 1815 et 1819 , firent apparaître trois camps ${ }^{(33)}$. Le premier était celui des notables libéraux qui, suivant le droit fondé en raison des Lumières et le constitutionnalisme de la Révolution française, voulaient une constitution sur le modèle de la Charte française. En ce qui concernait la composition de l'assemblée, on restait pragmatique: il suffisait d'ordonner que les conseillers généraux du département soient choisis parmi les plus gros contribuables, et d'étendre leur pouvoir, pour obtenir les États provinciaux correspondant aux besoins rhénans. Les villes rhénanes donnèrent leur avis par pétition. Mais le roi de Prusse interdit ce genre d'adresse ${ }^{(34)}$.

Les forces conservatrices, quant à elles, exigeaient le retour à la tradition des États provinciaux d'avant la domination française, avec des modifications : le critère de propriété l'emporterait sur la particule pour fonder les droits politiques et les ventes de biens nationaux ne seraient pas annulées. La noblesse renoncerait à sa décharge d'impôt et à son exigence que la haute fonction publique lui soit réservée. Cette position était, entre autres, celle de Joseph Görres - dont les écrits avaient porté la réputation au-delà du Rhin - et du baron von Stein, à la tête de l'administration de la rive gauche pendant la transition de 1813 et $1814^{(35)}$.

Il y avait un troisième camp qui se fit tout d'abord discret : celui des « vieux États » en faveur du retour à l'Ancien Régime, mené par de riches aristocrates du Bas-Rhin dont les chefs se rendirent en 1818 à Berlin pour gagner le soutien des cercles ultras gravitant autour du gouvernement. Mieux encore, ils parvinrent à convaincre le prince consort de se mettre à leur tête. L'étoile du grand réformateur Hardenberg commençait à pâlir, ce qui les favorisait. Ce dernier s'efforçait de couronner son œuvre en Prusse par une constitution instituant un seul parlement pour l'ensemble des États de la couronne, mais il échoua au début des années 1820, justement à cause de l'opposition des « vieux États » menée par le prince consort. Malgré l'abandon du projet de Hardenberg, le gouvernement prussien ne renonça pas à l'idée d'une représentation de l'ensemble du peuple, mais se contenta des États provinciaux jusqu'en 1848 dont ni la composition,

32 Gregor Bergerhausen, Die Großbürgerlichen Liberalen im Rheinischen Provinziallandtag, Cologne, Rheinland-Verlag, 1994, p. 46-49.

33 Karl-Georg FABER, Die Rheinlande zwischen Restauration und Revolution. Probleme der rheinischen Geschichte von 1814 bis 1848 im Spiegel der zeitgenössischen Publizistik, Wiesbaden, Steiner, 1966, p. 263-305.

34 À propos des discussions à Cologne et de leurs parallèles avec les autres villes du Rhin, voir Gisela Mettele, Bürgertum in Köln 1775-1870. Gemeinsinn und frei Association, Munich, Oldenbourg, 1998; Jürgen Herres, Köln in preußischer Zeit 1851-1871, Cologne, Greven Verlag, 2012, p. 60-64.

35 Nous nous bornons ici à indiquer les derniers titres de référence sur Görres et von Stein : MonIKA FINKLANG, Joseph Görres. Die Biographie, Paderborn, Schöningh, 2013, p. 145-191; Heinz DuchHARDT, Stein. Eine Biographie, Münster, Aschendorff, 2007, p. 365-369. 
ni les compétences ne répondaient aux vœux des libéraux rhénans: la nouvelle diète rhénane conservait la division par ordres ${ }^{(36)}$.

Attardons-nous un moment sur cette composition. Berlin avait commencé par attribuer les voix ainsi: trois sixièmes aux chevaliers, deux aux villes et un aux grands propriétaires terriens. Mais le gouvernement reconnut vite l'impossibilité de cette répartition, compte tenu de la société locale et de son héritage politique. On en vint aux dispositions suivantes. Le premier ordre était celui de la noblesse jadis dominante, représentée par les grandes familles du Saint-Empire et vingt-cinq chevaliers. Le second ordre était composé de vingt-cinq représentants des villes et le troisième de vingt-cinq propriétaires terriens. Considérons maintenant ces députés et constatons que même les villes et les « simples » propriétaires envoyèrent au parlement provincial des nobles qui, tous, disposaient d'immenses fortunes foncières. Quant à la position de la noblesse, deux citations l'illustrent: le baron Max von Loë déclarait encore en 1843 devant la diète qu'il parlait « en connaissance de cause: la législation n'avait pas compté en pourcentage, mais selon les principes de l'État; la seule modification selon le nombre ne peut être qu'en fonction de la quantité d'hectares que chacun possède, ou en fonction du nombre d'habitants. Mais quel Rhénan adopterait sérieusement cette dernière solution? " ${ }^{(37)}$ Le seigneur de Nordeck renchérit: « Le commerce et l'industrie ne valent rien pour gouverner ${ }^{(38)}$.

Dans les villes en plein essor, les libéraux de la bourgeoisie d'affaires étaient furieux de ce privilège accordé à la noblesse terrienne. Que les « chevaliers » aient vingt-cinq voix soulevait un concert de critiques chez les autres ordres; la population urbaine était représentée très en deçà de son importance numérique ${ }^{(39)}$. En somme, la composition de l'assemblée régionale fut l'objet de discussions infinies. Ses compétences aussi soulevaient le mécontentement. Elle était purement consultative et ne votait ni le montant des impôts ni leur répartition. Même pour une pétition, elle avait besoin de réunir une majorité des deux tiers. Les notables libéraux étaient profondément déçus et ne cessaient de comparer leur situation avec celle de la France et des États voisins du sud-ouest ${ }^{(40)}$.

Pris globalement, tout ceci montre que les années de transition ont été plutôt difficiles dans les anciens départements français passés à la Prusse. En outre, la situation économique n'était pas bonne: Berlin augmenta massivement les impôts pour payer son exorbitante dette de guerre et cela, davantage dans les nouveaux territoires qu'en vieille Prusse. En 1820, le taux d'imposition foncière augmenta de $20 \%$, tandis qu'un impôt sur la mouture des grains et l'abattage des bêtes était introduit; boulangers et charcutiers des villes durent payer une taxe supplémentaire sur leurs produits ${ }^{(41)}$.

36 Joachim Stephan, Der Rheinische Provinziallandtag 1826-1840, Cologne, Rheinland-Verlag, 1991, p. 21-25.

37 Ibid., p. 39.

38 Ibid.

39 Gustav Croon, Der Rheinische Provinziallandtag bis zum Jahre 1874, Düsseldorf, Voß \& Co, 1918, p. 29-34.

40 R. Sснӥтz, Preußen und die Rheinlande (note 13), p. 246.

41 J. Herres, Köln in preußischer Zeit 1851-1871 (note 34), p. 69. 
Surtout, une crise alimentaire séculaire s'était déclarée au moment où les Prussiens avaient pris le pouvoir. En avril 1815, le volcan Tambora entrait en éruption dans le sud du Pacifique, éruption que les spécialistes considèrent comme la plus forte des dix mille dernières années ${ }^{(42)}$. Des quantités de cendres et de particules restèrent dans la stratosphère tout autour de la terre et gênèrent le passage des rayons du soleil. La conséquence fut ce qu'on appelle un " hiver volcanique », pendant lequel les températures moyennes baissèrent de trois à quatre degrés. Cet accident provoqua un refroidissement de plusieurs années sur l'ensemble du globe, de mauvaises récoltes et des famines. Les contemporains ne pouvaient imaginer la raison de cette catastrophe climatique. Mais on rend volontiers le gouvernement responsable de ce qui ne va pas, surtout lorsqu'il ne se montre pas à la hauteur de la crise. Les livraisons de grain commandées par l'État furent trop longues à arriver et restèrent insuffisantes ${ }^{(43)}$. Laissons le mot de la fin à un paysan, dont Sulpiz Boisserée, collectionneur d'art à Cologne, rapporta le propos dans son journal intime du 8 juin 1815. Ce que les occupants d'outreRhin n'avaient pas réussi en vingt ans, dit-il, « les Prussiens l'ont fait en six mois: nous rendre les Français agréables " ${ }^{(4)}$.

\section{Traduit de l'allemand par Ségolène Plyer}

\section{Zusammenfassung}

Die linksrheinischen Departements des napoleonischen Empires wurden 1815 auf dem Wiener Kongress dem Königreich Preußen zugeteilt. Im Rheinland hielt sich die Begeisterung darüber, nun preußischer Untertan zu werden, in engen Grenzen. Der Beitrag fokussiert drei neuralgische Punkte in der ersten Phase der Restauration: Die preußische Beamtenpolitik, die Frage der Nationalgüter und die Auseinandersetzungen um die politische Partizipation. Während die Beamtenpolitik und die Frage der Nationalgüterverkäufe weitgehend konfliktfrei geregelt wurden, weil Preußen hier wenig veränderte, kam es bezüglich der Frage der politischen Partizipation zu heftigen Kontroversen. Dies vor allem, weil der König das Versprechen eine Verfassung zu erlassen, nicht einlöste.

\section{Résumé}

Entre 1794 et 1814, la rive gauche du Rhin changea deux fois de régime politique: sous la Révolution puis au congrès de Vienne. À la fin du XIX siècle, l'historiographie allemande la considéra comme le bastion avancé de la germanité, mais l'analyse historique récente retire une impression plus mitigée des traces écrites laissées par les cadres de l'administration et de la justice ou les bourgeois, notamment dans les territoires attribués à la Prusse en 1815. L'attachement à l'État ne se définissant alors pas en termes nationaux, les Français avaient été jugés à leur comportement, notamment aux

42 Wolfgang Behringer, Kulturgeschichte des Klimas. Von der Eiszeit bis zur globalen Erwärmung, Munich, Beck, 2007, p. 217 sq.

43 K.-G. FABer, Die Rheinlande zwischen Restauration und Revolution (note 33), p. 287.

44 Sulpiz BoisseréE, Tagebücher (1808-1854), édités par Hans-Joachim Weitz, 5 volumes, Darmstadt, Roether, 1978-1995, vol. 1, p. 185. 
réformes qu'ils avaient introduites (modernisation juridique, vente de biens nationaux) qui trouvèrent un écho favorable dans la population. Le passage à l'administration prussienne en 1815 ne se fit pas sans controverses, qui poussèrent le nouveau pouvoir à entériner les transferts de propriété et à ne pas restaurer complètement l'ancienne prééminence de la noblesse. Des difficultés économiques dues au coût des guerres contre Napoléon et aux mauvaises récoltes achevèrent de rendre le retour à l'Allemagne moins attractif que le souvenir de l'annexion française. 